\title{
Hydrogel-based piezoresistive sensor for the detection of ethanol
}

\author{
Jan Erfkamp, Margarita Guenther, and Gerald Gerlach \\ Solid-State Electronics Laboratory, Technische Universität Dresden, Dresden, Germany \\ Correspondence: Jan Erfkamp (jan.erfkamp@tu-dresden.de)
}

Received: 18 January 2018 - Revised: 6 March 2018 - Accepted: 9 March 2018 - Published: 4 April 2018

\begin{abstract}
This article describes a low-cost sensor for the detection of ethanol in alcoholic beverages, which combines alcohol-sensitive hydrogels based on acrylamide and bisacrylamide and piezoresistive sensors. For reproducible measurements, the reversible swelling and deswelling of the hydrogel were shown via microscopy. The response time of the sensor depends on the swelling kinetics of the hydrogel. The selectivity of the hydrogel was tested in different alcohols. In order to understand the influence of monomer and crosslinker content on the swelling degree and on the sensitivity of the hydrogels, gels with variable concentrations of acrylamide and bisacrylamide were synthesized and characterized in different aqueous solutions with alcohol contents. The first measurements of such hydrogel-based piezoresistive ethanol sensors demonstrated a high sensitivity and a short response time over several measuring cycles.
\end{abstract}

\section{Introduction}

The ethanol concentration is one of the essential parameters of process analytics in the brewing industry. Standard methods used such as high-performance liquid chromatography (HPLC) (Castellari et al., 2001) or the enzymatic determination of alcohol via UV-VIS spectroscopy (Korb, 2003) are costly and expensive, especially for small breweries. In comparison, manual measurement methods like hydrometers often have a poor resolution, and the accuracy of the measurement depends on the care and expertise of the operator. Furthermore, the measurements depend strongly on the sample temperature (Osorio et al., 2008). As a consequence, breweries often need a better laboratory method for the detection of ethanol, especially considering the strict European Union laws on the labeling of alcoholic beverages (Lachenmeier et al., 2010). In this paper, a piezoresistive silicon sensor is presented that makes use of the ethanol-dependent swelling of hydrogels. This principle has a great potential for costeffective and miniaturized sensors that are capable of inline measurements.

Hydrogels are "intelligent" hydrophilic polymer networks that show swelling properties depending on various stimuli, such as $\mathrm{pH}$, salt concentration or temperature (Guenther et al., 2014). In combination with piezoresistive pressure sen- sors (which measure the resulting swelling pressure), novel sensors for biotechnology, environmental and process analysis can be realized (Binder et al., 2017; Schmidt et al., 2016; Jorsch et al., 2016).

Ethanol-dependent swelling has already been demonstrated for hydrogels based on poly ( $N$-isopropylacrylamide) (Arndt et al., 2000; Mukae et al., 1993) and polyacrylic acid (Nishiyama and Satoh, 2000). In this work, based on polyacrylamide-bisacrylamide hydrogels, a piezoresistive hydrogel sensor will be presented, which is inexpensive and highly sensitive to ethanol, e.g., in alcoholic beverages. The alcohol concentration change leads to a reversible swelling or shrinking of the hydrogel. The swelling pressure on the bending plate of the used pressure sensor leads to a piezoresistive change in resistance and, via a Wheatstone bridge circuit, to a change in the measuring signal, which depends proportionally on the ethanol concentration.

\section{Materials and methods}

\subsection{Synthesis of polyacrylamide-bisacrylamide hydrogels}

For the synthesis of the hydrogels, $10 \mathrm{vol} \%(0.32 \mathrm{mmol}$, $22.61 \mathrm{mg}$ ) acrylamide (Aam, Sigma Aldrich) and $0.44 \mathrm{~mol} \%$ 


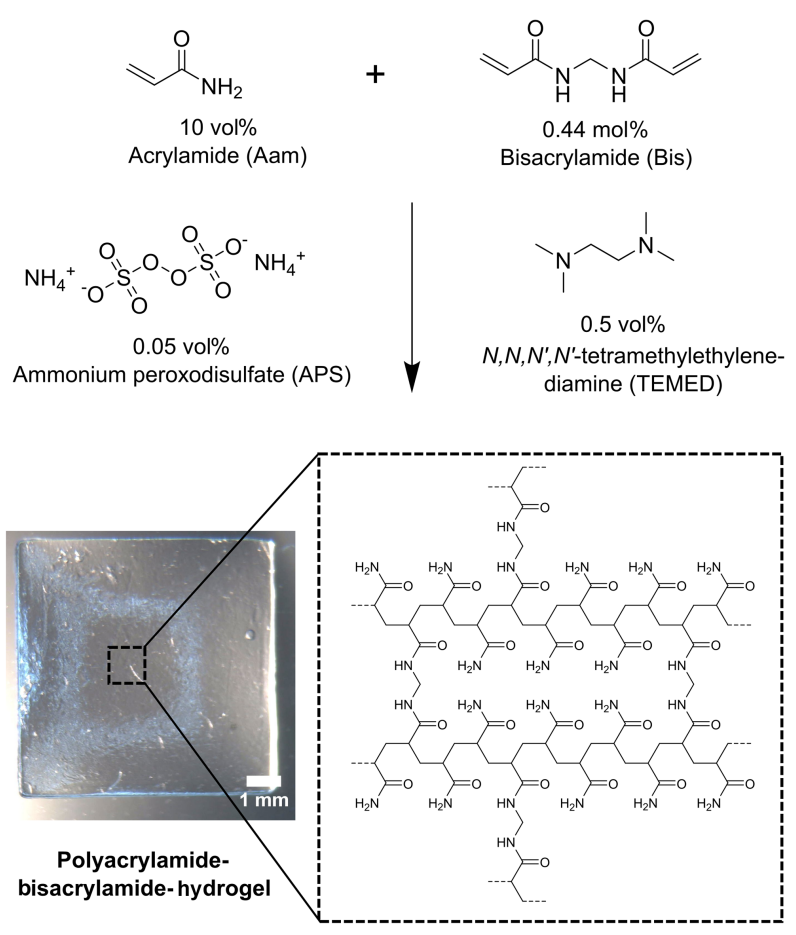

Figure 1. Polymerization of ethanol-sensitive polyacrylamidebisacrylamide hydrogels.

(1.39 $\mu \mathrm{mol}, 0.21 \mathrm{mg}$ ) bisacrylamide (Bis, Carl Roth) were dissolved in distilled water and degassed with nitrogen for $5 \mathrm{~min}$. In the syntheses with different acrylamide and bisacrylamide concentrations, the ratios were varied accordingly. Subsequently, the polymerization was initiated by adding $0.05 \mathrm{vol} \%(0.87 \mu \mathrm{mol}, 0.20 \mathrm{mg})$ ammonium peroxodisulfate (Sigma Aldrich) and $0.5 \mathrm{vol} \%(1.0 \mu \mathrm{L}) N, N, N^{\prime}, N^{\prime}$ tetramethylethylenediamine (Carl Roth). The total volume of a synthesis approach was $200 \mu \mathrm{L}$. Each $30 \mu \mathrm{L}$ of the pregel was placed in a plastic wafer mold $(5.32 \mathrm{~mm} \times 5.32 \mathrm{~mm} \times$ $0.76 \mathrm{~mm}$, Fluoroware) and polymerized there for at least $6 \mathrm{~h}$. To remove unreacted monomers from the synthesized polymers, the hydrogels were washed in distilled water for 3 days. The mechanism of the polymerization is shown in Fig. 1.

\subsection{Swelling studies in alcohol-water mixtures}

After the synthesis, the hydrogels were initially conditioned 5 times in a corresponding alcohol solution. Subsequently, the hydrogels were washed in distilled water and the initial length of the hydrogel $l_{0}$ was determined after $24 \mathrm{~h}$ via microscopy. After the preparation of an ethanol-water mixture with a corresponding volume concentration of ethanol, the hydrogels were incubated in this solution for $24 \mathrm{~h}$. Following the determination of the length of the hydrogel $l$ via microscopy, the solutions were changed again. For the swelling kinetics, the length of the hydrogel $l$ was determined after a (a)

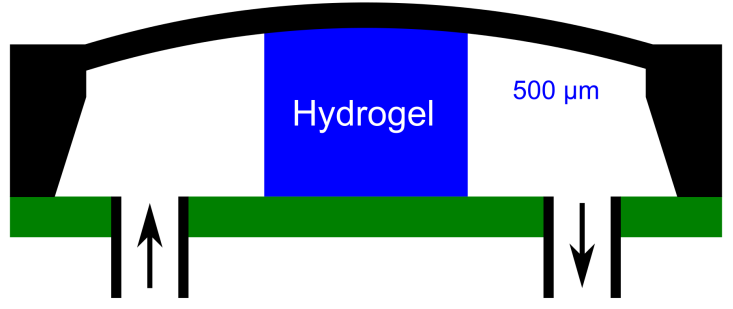

(b)

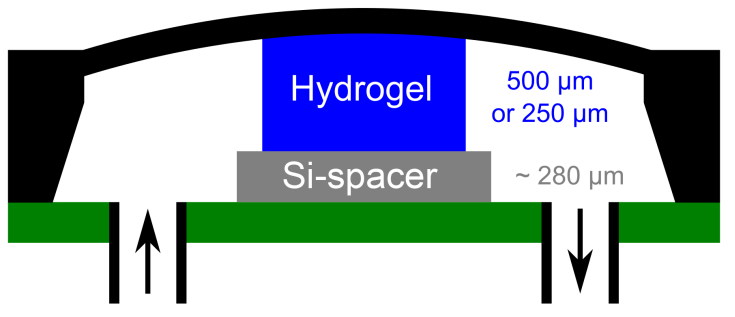

Figure 2. Sensor setups: (a) $500 \mu \mathrm{m}$ thick hydrogel body after synthesis without silicon spacer, (b) 250 or $500 \mu \mathrm{m}$ thick hydrogel body after synthesis with silicon spacer (thickness ca. $280 \mu \mathrm{m}$ ).

defined time. All experiments were performed at room temperature. From the results, the swelling degree $S$ of the hydrogel was calculated as a relative change in length (Bai et al., 2013):

$S=\frac{l-l_{0}}{l_{0}} \times 100 \%$.

\subsection{Sensor design}

At first, for applications in a sensor, defined hydrogel layers had to be produced. For this, a plastic spacer (thickness of 250 and $500 \mu \mathrm{m}$, respectively) was fixed between two glass slides and the pregel solution was filled into the resulting cavity (volume ca. $250 \mu \mathrm{L}$ ). After the polymerization, the gel was washed in distilled water for 3 days. Circular hydrogels with a diameter of $1.5 \mathrm{~mm}$ were punched out of the gel. In addition, various sensor setups were tested (Fig. 2). First, a circular hydrogel with a thickness of $500 \mu \mathrm{m}$ after synthesis was put in the center of the cavity of the pressure sensor (EPCOS, C41-Series, $5.0 \mathrm{~mm} \times 5.0 \mathrm{~mm} \times 0.4 \mathrm{~mm}$ ). In a further approach, a silicon spacer (thickness ca. $280 \mu \mathrm{m}$ ) was fixed by the use of cyanoacrylate on a circuit board. The circular hydrogels with a thickness of 250 or $500 \mu \mathrm{m}$ after synthesis were fitted on the silicon spacer. Now, in all cases, the pressure sensor was fixed by means of cyanoacrylate on a circuit board. After bonding the sensor chip to the circuit board, connection cables to the read-out unit (Fluke 45 Multimeter) were soldered. In order to feed and analyze the ethanol-water mixtures to the hydrogel sensor, an inlet and outlet hose was fixed on the circuit board using cyanoacrylate and two-component epoxy resin. The resulting sensor (Fig. 3) was connected to a peristaltic pump at a constant flow 


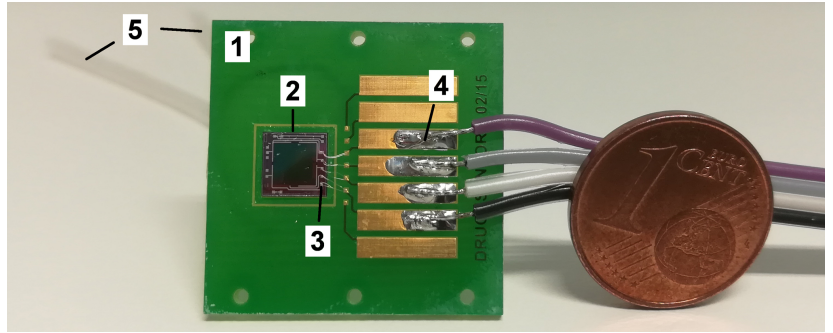

Figure 3. Hydrogel-based piezoresistive sensor for the detection of ethanol; (1) circuit board, (2) pressure sensor chip with hydrogel filled in the cavity, (3) bonding islands with bonded gold wires, (4) soldered connection cables, (5) inlet and outlet hoses to the pump.

of $0.2 \mathrm{~mL} \mathrm{~min}^{-1}$. For the sensor measurements, the ethanolwater mixtures were changed every $30 \mathrm{~min}$.

\section{Results and discussion}

\subsection{Reversibility of hydrogel swelling}

The reproducibility of the alcohol sensor is crucial for applications in process analysis. Based on the reversible swelling and deswelling process, hydrogels are excellently suited for applications in sensor technology (Fig. 4). With the change from 0 to $50 \mathrm{vol} \%$ ethanol, the hydrogel deswells by about $35 \%$. Changing back the ethanol concentration to $0 \mathrm{vol} \%$ causes the hydrogels to swell to their initial size. This behavior could also be reproduced during subsequent solvent changes. Stimuli-responsive hydrogels are already known for reversible swelling, as shown for temperature (Guenther et al., 2006), pH (Liu et al., 2016) and salt-sensitive (Izawa et al., 2009) hydrogels.

To understand the reversible swelling mechanism of polyacrylamide-bisacrylamide hydrogels in ethanol, it is important to know why these hydrogels have a sensitivity to ethanol. Fadouloglou et al. used ethanol to dry out polyacrylamide hydrogels, which are the standard system for gel electrophoresis of proteins, reproducibly after electrophoresis and observed a uniform shrinking of the gel after $20 \mathrm{~min}$ in $95 \mathrm{vol} \%$ ethanol (Fadouloglou et al., 2000). However, the authors have not given any explanation for this effect. Çaykara and Doğmuş characterized swelling properties of polyacrylamide gels in different alcoholic mixtures. They explained the shrinking of the hydrogels in alcohol-water mixtures with attractive interactions between alcohol and water. As a result, the free energy for the polymer-polymer contact increased, the polymer chains interact stronger with each other and the hydrogel deswells (Çaykara and Doğmuş, 2004). The sensitivity of hydrogels to ethanol was studied by using different hydrogel systems. Xu et al. polymerized photonic crystals based on hydroxyethyl methacrylate hydrogels. They explained the swelling of the hydrogel from
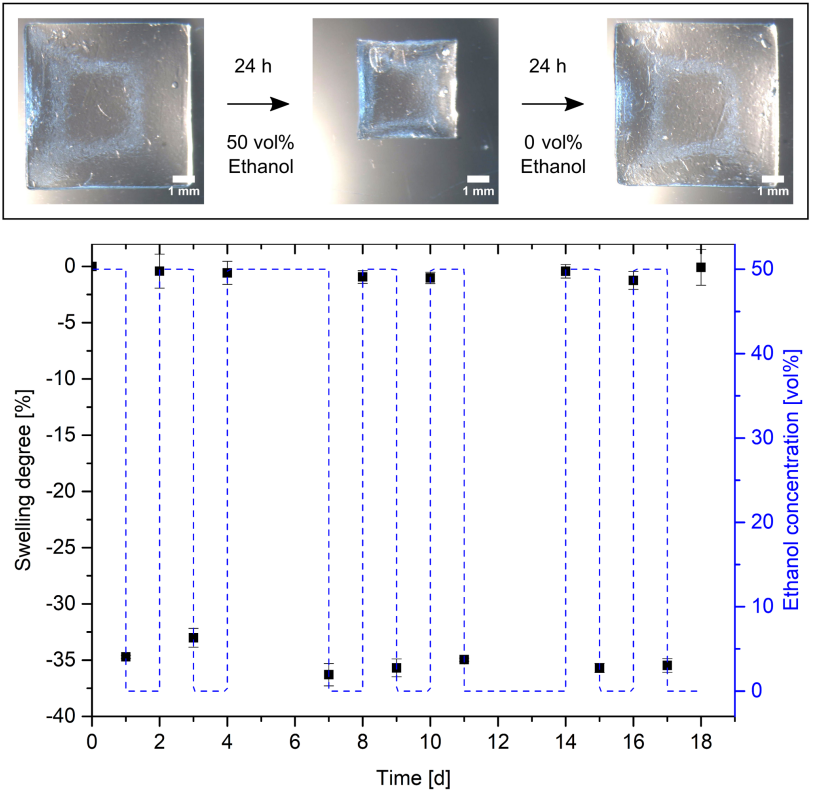

Figure 4. Reversible hydrogel swelling versus ethanol concentration (hydrogel: $10 \mathrm{vol} \%$ Aam and $0.44 \mathrm{~mol} \% \mathrm{Bis}$ ).

pure water to $40 \mathrm{vol} \%$ ethanol with an increase in free energy in the polymer network in the presence of ethanol $(\mathrm{Xu}$ et al., 2008). Guenther and Gerlach described the ethanol sensitivity of poly( $N$-isopropylacrylamide) hydrogels with the positive viscosity coefficient of ethanol. Thereby, the hydrophobic interactions in the gel tend to enhance at low ethanol concentrations, which leads to stronger polymerpolymer interactions and a deswelling of the polymer system (Guenther and Gerlach, 2009). All these effects can influence the deswelling of the characterized polyacrylamidebisacrylamide hydrogel system in alcoholic solutions.

\subsection{Swelling kinetics in different alcohols}

The hydrogels are sensitive not only to ethanol but to other alcohols such as methanol and isopropanol. The sensitivity to ethylene glycol, however, is very low (Fig. 5). The polarity has a significant influence on the interaction of the solvent with the gel. The more polar the alcohol, the lower the alcohol-hydrogel interactions and the swelling degree of the gel. Ethylene glycol has two polar hydroxyl groups $\left(\Delta l / l_{0}=\right.$ $1.5 \%)$, and the other alcohols have only one polar hydroxyl group. As a result, ethylene glycol can form more hydrogen bonds than the other alcohols. The different length of the hydrophobic alkyl groups in the ethanol $\left(\Delta l / l_{0}=35.4 \%\right)$, isopropanol $(36.8 \%)$ and methanol $(34.0 \%)$ seem to slightly influence the degree of swelling. The polarity also has a considerable influence on the solubility of a polymer. The solubility of polyacrylamide, which is the main component of the hydrogel, significantly influences the physical properties of the hydrogel, like the solubility behavior. Polyacrylamide 


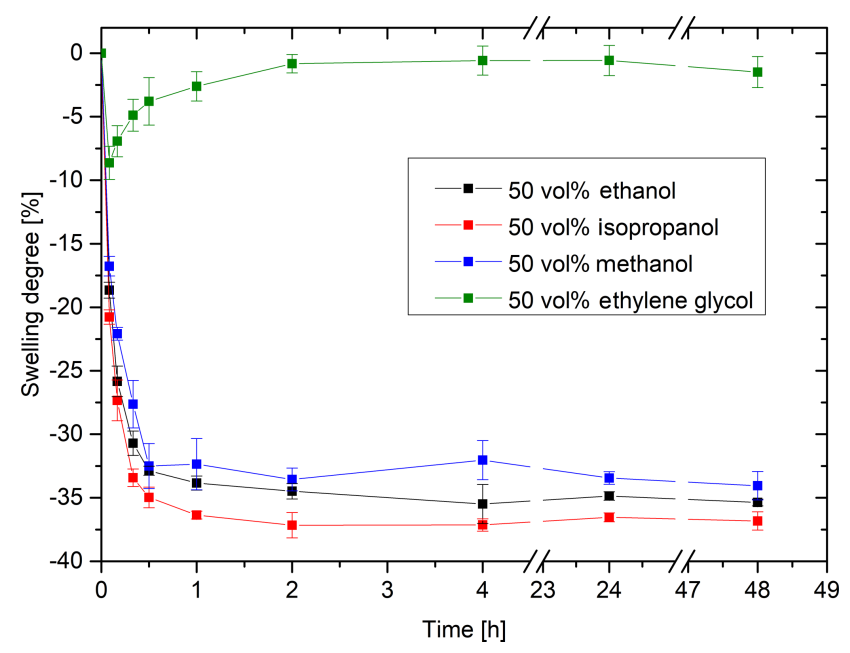

Figure 5. Swelling kinetics in ethanol, isopropanol, methanol and ethylene glycol (hydrogel: 10 vol\% Aam and $0.44 \mathrm{~mol} \%$ Bis).

is soluble in water or ethylene glycol. However, the polymer is insoluble in alcohols (Cheremisinoff, 1997). In water or ethylene glycol, the polymer chains will interact with the solvent and the hydrogel swells. With the change to alcoholic solutions, the polymer chains will not interact with the solvent. As a consequence, the functional groups of the gel will interact with each other and the hydrogel deswells in alcoholic solutions.

Another effect affecting the swelling behavior is the viscosity of the solvent. Compared to the other alcohols, ethylene glycol has an absolute viscosity that is 10 times higher (Smallwood, 1996). The diffusion coefficient decreases with increasing absolute viscosity according to the Stokes-Einstein equation, whereby the molecules diffuse more slowly into the hydrogels.

The swelling kinetics also shows that the hydrogels are almost completely swollen after just $1 \mathrm{~h}$. However, it must be considered that the size of the gel has an influence on the swelling kinetics. Much smaller hydrogels were used in the sensor application (circular hydrogels with a total volume of $3.5 \mathrm{~mm}^{3}$ ) than in free-swelling experiments (squared hydrogels with a total volume of $30 \mathrm{~mm}^{3}$ ). The diffusion path of the solvent and, thereby, the diffusion time and the response time of the hydrogel were decreased. This indicates a much faster response time in sensor applications. The cross-sensitivity to other alcohols is not a problem for alcohol analysis applications because alcoholic beverages contain only ethanol.

\subsection{Sensitivity of hydrogel swelling in different alcohols}

It is often required for alcohol percentage determination that the sensor characteristic curve should be as linear as possible in the range of 0 to $50 \mathrm{vol} \%$ ethanol. For this reason, the influence of the alcohol concentration on the swelling behavior of the hydrogels was investigated (Fig. 6). All tested

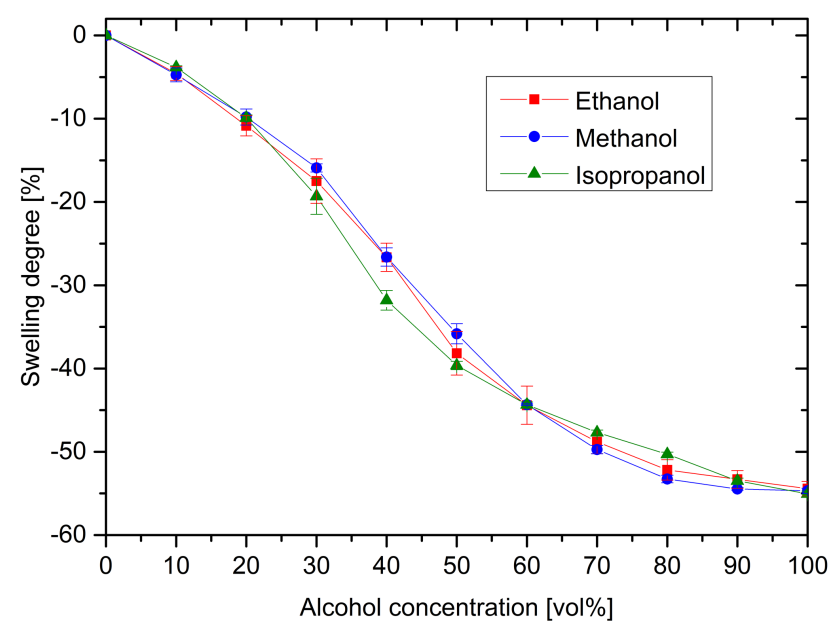

Figure 6. Swelling degree at different alcohol concentrations of ethanol, methanol and isopropanol (hydrogel: $10 \mathrm{vol} \%$ Aam and $0.44 \mathrm{~mol} \%$ Bis).

alcohols showed similar sensitivities over the entire concentration range. Even at low alcohol concentrations, swelling processes were detectable. This indicates a low detection limit, which could be of particular interest for alcohol detection in the brewing industry. The dependence of the hydrogel swelling degree on the alcohol concentration is quasi-linear over a large concentration range.

A quasi-linear ethanol sensitivity was also observed for hydrogels based on acrylamide-co-itaconic acid (Çaykara and Doğmuş, 2004). In comparison, $\operatorname{poly}(N-$ isopropylacrylamide) hydrogels deswell in a range of 0 to $20 \mathrm{vol} \%$. Almost no change in the swelling degree was observed from 20 to $40 \mathrm{vol} \%$, before the hydrogels swell again within a range of 40 to $100 \mathrm{vol} \%$ (Richter, 2002). This so-called co-nonsolvency effect (Liu et al., 2015; Winnek et al., 1992) was described for other hydrogel systems like poly(acryloyl- $L$-proline methyl ester) gels (Hiroki et al., 2001). This difference can be explained by the different chemical structure and the resulting different interactions of the solvent with the hydrogel. However, this nonlinear swelling behavior is unfavorable compared to the quasi-linear behavior of the polyacrylamide hydrogels for sensor applications.

\subsection{Influence of the acrylamide and bisacrylamide concentration}

The composition of the hydrogel influences the swelling behavior and, thus, also the sensitivity of the sensor. For that purpose, the influence of the acrylamide volume concentration on the swelling behavior of the hydrogels was determined at first (Fig. 7). With increasing acrylamide concentration the sensitivity of the hydrogel decreases because the chain length of the polymer increases, which results in over- 


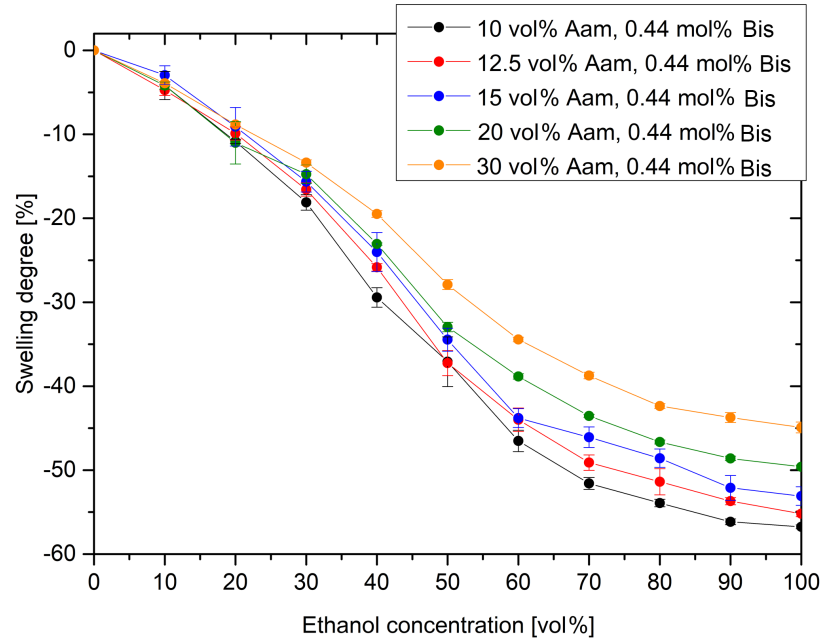

Figure 7. Influence of the acrylamide concentration on the swelling degree of the hydrogels.

laps and entanglements of polymer chains within the hydrogel. The resulting physical crosslinking reduces the swelling degree (Wack, 2006) but also increases the mechanical stability of the hydrogel. Lower concentrations of acrylamide ( $\leq 7.5 \mathrm{vol} \%$ Aam) lead to a deterioration of the mechanical properties so that these gels cannot longer be used for sensory purposes.

In addition to the monomer acrylamide, the crosslinker bisacrylamide also influences the swelling behavior (Fig. 8). The lower the concentration of the crosslinker bisacrylamide, the larger the shrinking of the hydrogels. A low proportion of crosslinkers leads to fewer crosslinking points and, thus, to a larger mesh size in the hydrogel (Wack, 2006). This leads to a higher sensitivity, but also to a reduction in the mechanical stability of the gel. At lower concentrations of bisacrylamide ( $\leq 0.25 \mathrm{~mol} \% \mathrm{Bis}$ ), the hydrogel becomes mechanically so unstable that it is no longer usable. Therefore, for sensor applications, the hydrogels must be adjusted so that the highest possible sensitivity and the simultaneous mechanical stability are present.

\subsection{Piezoresistive ethanol sensor}

The ethanol-induced swelling of the hydrogel causes sufficiently large forces to deform the bending plate of the pressure sensor, whereby an output voltage $U_{\text {out }}$ is measurable (Fig. 9). This can be used to study the reproducibility of the swelling of the hydrogel over several swelling cycles. As expected, the response time of the sensors amounting to a few minutes is shorter than of hydrogels in free-swelling mode. The response times of the sensors could be further reduced by using porous hydrogels (Franke et al., 2017).

A drift of the sensor signal was observed. This effect can be minimized by improving the sensor design. In the swelling process, an overshooting of the sensor was detected

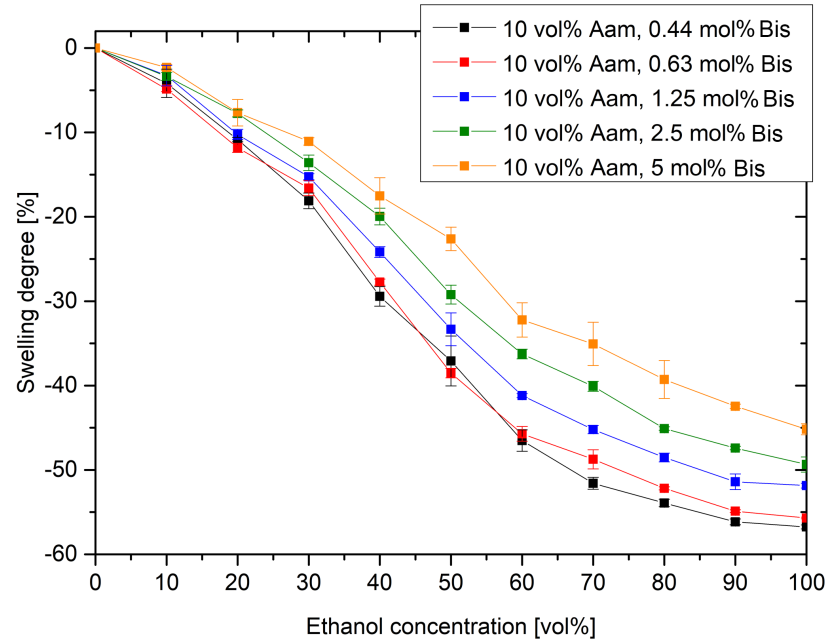

Figure 8. Influence of the bisacrylamide concentration on the swelling degree of the hydrogels.

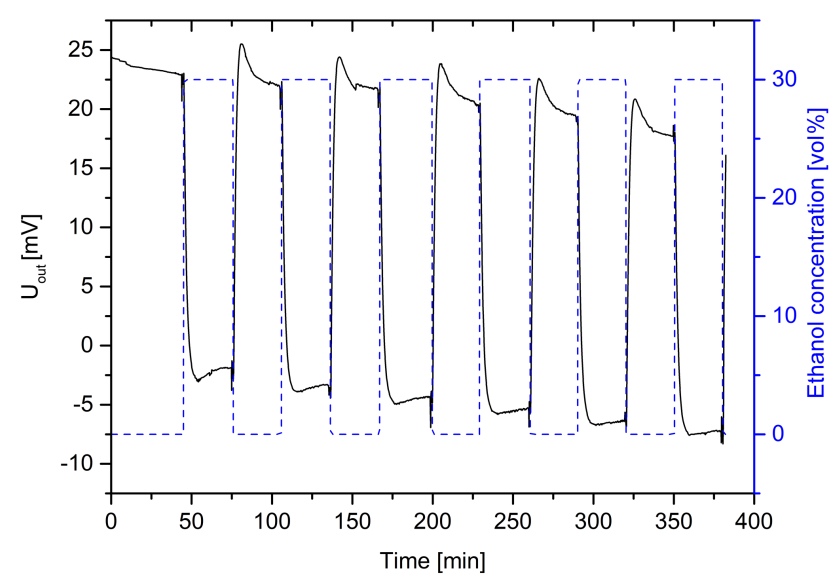

Figure 9. Output voltage of the piezoresistive hydrogel-based pressure sensor for cyclic changes in the ethanol concentration (hydrogel: 10 vol\% Aam and $0.44 \mathrm{~mol} \%$ Bis; sensor setup: $250 \mu \mathrm{m}$ hydrogel with silicon spacer).

by changing the ethanol concentration from 30 to $0 \mathrm{vol} \%$. Due to the lower crosslinker concentration, the hydrogel is very soft after swelling and the uptake of water. As a result, the opposing forces of the bending plate press water out of the gel, which leads to the overshooting of the sensor. The mechanical properties of the hydrogel can be improved by higher crosslinker concentrations. In this way, the overshooting should be avoided. When changing the ethanol concentration from 0 to $30 \mathrm{vol} \%$, signal changes of about $25 \mathrm{mV}$ occurred. Due to the large signal change, it is to be expected that even low ethanol concentrations, like in beer (ca. $5 \mathrm{vol} \%$ ethanol), can be detected.

Furthermore, different sensor setups were tested (Fig. 10). The cavity of the pressure sensor has a height of $390 \mu \mathrm{m}$ (EPCOS AG, 2017). In order to ensure the contact between 


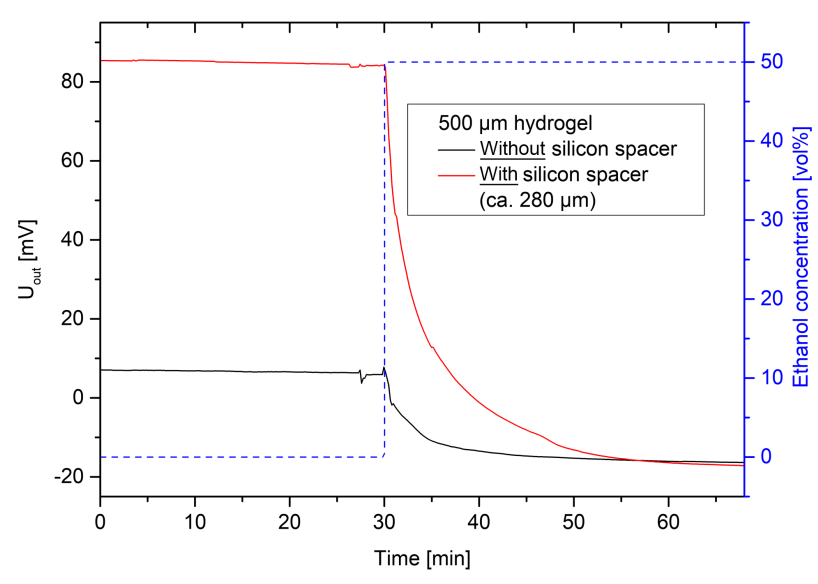

Figure 10. Influence of the sensor setup on the output signal (hydrogel: 10 vol\% Aam and $0.44 \mathrm{~mol} \%$ Bis).

the hydrogel and the bending plate during gel shrinking, the largest possible deflection of the bending plate should be achieved. For that reason and because of the viscoelastic properties of the gel, it is necessary to use slightly thicker hydrogels without damaging the bending plate. A thicker hydrogel leads to a larger predeflection of the bending plate of the pressure sensor. The thicker the gel is, the longer a contact between the hydrogel and the bending plate exists, which means a larger concentration range should be detectable.

The predeflection of the sensor can be defined as the output voltage in pure water. The sensor with a $500 \mu \mathrm{m}$ thick hydrogel using a silicon spacer had the largest deflection $(85 \mathrm{mV})$ and the similar sensor without a silicon spacer had the least deflection $(7 \mathrm{mV})$. When changing the ethanol concentration from 50 to $0 \mathrm{vol} \%$, signal changes of about $101 \mathrm{mV}$ for the hydrogel with spacer and $23 \mathrm{mV}$ for the one without spacer were detected.

As already discussed, the response time of the sensor depends largely on swelling kinetics and, thus, on the size of the hydrogel. Since the hydrogels have the same dimensions in both sensor constructions, no significant differences in the response times were observed.

After the shrinking of the hydrogel in the sensor, both sensor setups have nearly the same output voltage (ca. $-17 \mathrm{mV}$ ). This might be caused by the lack of contact between the hydrogel and the bending plate. In this case, the detection of higher ethanol concentrations would not be possible. However, the sensor setup using a silicon spacer was the favorable setup due to the larger predeflection of the bending plate and the change of the output signal being almost 5 times higher. Instead of a silicon spacer, hydrogels with a thickness of more than $500 \mu \mathrm{m}$ could also be used. However, thicker hydrogels could lead to longer response times of the hydrogel and the sensor.

Another alternative, the direct polymerization of the hydrogel (with a volume of $12.5 \mu \mathrm{L}$ ) in the cavity of a sen- sor, cannot be used. After swelling, the complete cavity was filled with hydrogel. The analysis solution cannot be pumped though the sensor. Also, when increasing the constant flow from 0.2 to $1.0 \mathrm{~mL} \mathrm{~min}^{-1}$, the pump resistance of the hydrogel in the cavity was too high.

\section{Summary}

In this paper, the suitability of acrylamide-bisacrylamide hydrogels for the detection of ethanol in aqueous solutions was demonstrated. The swelling pressure of the hydrogel was measured by piezoresistive pressure sensors. It was shown that hydrogels with 10 vol\% acrylamide and $0.44 \mathrm{~mol} \%$ bisacrylamide led to sufficiently high sensitivities, so that such sensors can be used in situ in the production of alcoholic beverages such as beer or high-percentage alcoholic drinks. The composition has an influence on the sensitivity and the mechanical stability of the hydrogel. The hydrogel also showed a similar sensitivity to methanol and isopropanol. The characteristics of the sensors demonstrated a nearly linear course in a large concentration range, which is a great advantage for the use as ethanol sensors in alcohol production. The inline capability of the sensor, its high sensitivity, the good linearity, the short response time of the sensor of a few minutes and the cost-effective production make this sensor concept particularly interesting for industrial applications.

Data availability. According to the guidelines of the Deutsche Forschungsgemeinschaft (DFG), the presented data in this publication are stored in an internal system. All measurement data are not publicly available and can be accessed from the authors upon request.

Competing interests. The authors declare that they have no conflict of interest.

Special issue statement. This article is part of the special issue "Dresden Sensor Symposium 2017". It is a result of the Dresden Sensor Symposium 2017, Dresden, Germany, 4-6 December 2017.

Acknowledgements. The authors would like to thank the Deutsche Forschungsgemeinschaft (DFG) for financial support within the Research Training Group "Hydrogel-based Microsystems" (GRK 1865).

Edited by: Udo Weimar

Reviewed by: two anonymous referees 


\section{References}

Arndt, K.-F., Kuckling, D., and Richter, A.: Application of sensitive hydrogels in flow control, Poly. Advan. Technol., 11, 496-505, https://doi.org/10.1002/10991581(200008/12)11:8/12<496::AID-PAT996>3.0.CO;2-7, 2000.

Bai, W., Gariano, N. A., and Spivak, D. A.: Macromolecular Amplification of Binding Response in Superaptamer Hydrogels, J. Am. Chem. Soc., 135, 6977-6984, https://doi.org/10.1021/ja400576p, 2013.

Binder, S., Krause, A. T., Voit, B., and Gerlach, G.: Bisensitive Hydrogel With Volume Compensation Properties for Force Compensation Sensors, IEEE Sens. Lett., 1, 4501004, https://doi.org/10.1109/LSENS.2017.2774922, 2017.

Castellari, M., Sartini, E., Spinabelli, U., Riponi, C., and Galassi, S.: Determination of Carboxylic Acids, Carbohydrates, Glycerol, Ethanol, and 5-HMF in Beer by HighPerformance Liquid Chromatography and UV-Refractive Index Double Detection, J. Chromatogr. Sci., 39, 235-238, https://doi.org/10.1093/chromsci/39.6.235, 2001.

Çaykara, T. and Doğmuş, M.: The effect of solvent composition on swelling and shrinking properties of poly(acrylamideco-itaconic acid) hydrogels, Eur. Polym. J., 40, 2605-2609, https://doi.org/10.1016/j.eurpolymj.2004.06.024, 2004.

Cheremisinoff, N. P. (Eds.): Handbook of Engineering Polymeric Materials, Marcel Dekker Inc., New York, USA, 1997.

EPCOS AG: Pressure sensors - C41 series, available at: https://de. tdk.eu/inf/57/ds/c41_gauge.pdf, last access: 19 December 2017.

Fadouloglou, V. E., Glykos, N. M., and Kokkinidis M.: A Fast and Inexpensive Procedure for Drying Polyacrylamide Gels, Anal. Biochem., 287, 185-186, https://doi.org/10.1006/abio.2000.4852, 2000.

Franke, D., Binder, S., and Gerlach, G.: Performance of FastResponsive, Porous Crosslinked Poly(N-Isopropylacrylamide) in a Piezoresistive Microsensor, IEEE Sens. Lett., 1, 1500904, https://doi.org/10.1109/LSENS.2017.2773626, 2017.

Guenther, M. and Gerlach G.: Hydrogels for Chemical Sensors, in: Hydrogel Sensors and Actuators - Engineering and Technology, Springer Series on Chemical Sensors and Biosensors 6, edited by: Gerlach, G. and Arndt, K.-F., Springer-Verlag, BerlinHeidelberg, 165-195, https://doi.org/10.1007/978-3-540-756453_5, 2009 .

Guenther, M., Gerlach, G., Kuckling, D., Kretschmer, K., Corten, C., Weber, J., Sorber, J., Suchaneck, G., and Arndt, K.-F.: Chemical sensors based on temperature-responsive hydrogels, in: Smart Structures and Materials 2006: Smart Sensor Monitoring Systems and Applications, San Diego, California, United States, Proc. SPIE, 6167, 61670T, https://doi.org/10.1117/12.657126, 2006.

Guenther, M., Wallmersperger, T., and Gerlach, G.: Piezoresistive Chemical Sensors Based on Functionalized Hydrogels, Chemosensors, 2, 145-170, https://doi.org/10.3390/chemosensors2020145, 2014.

Hiroki, A., Maekawab, Y., Yoshidab, M., Kubotac, K., and Katakaia R.: Volume phase transitions of poly(acryloyl-1-proline methyl ester) gels in response to water-alcohol composition, Polymer, 42, 1863-1867, https://doi.org/10.1016/S0032-3861(00)00416$\mathrm{X}, 2001$.
Izawa, H., Kaneko, Y., and Kadokawa, J.: Unique gel of xanthan gum with ionic liquid and its conversion into high performance hydrogel, J. Mater. Chem., 19, 6969-6972, https://doi.org/10.1039/b916864h, 2009.

Jorsch, C., Schmidt, U., Ulkoski, D., Scholz, C., Guenther, M., and Gerlach, G.: Implantable biomedical sensor array with biocompatible hermetic encapsulation, J. Sens. Sens. Syst., 5, 229-235, https://doi.org/10.5194/jsss-5-229-2016, 2016.

Korb, C.: Entwicklung enzymatischer Screeningverfahren, Ph.D. thesis, Martin-Luther-Universität Halle-Wittenberg, Germany, 2003.

Lachenmeier, D. W., Godelmann, R., Steiner, M., Ansay, B., Weigel, J., and Krieg G.: Rapid and mobile determination of alcoholic strength in wine, beer and spirits using a flow-through infrared sensor, Chem. Cent. J., 4, 5, https://doi.org/10.1186/1752153X-4-5, 2010.

Liu, B., Wang, J., Ru, G., Liu, C., and Feng, J.: Phase Transition and Preferential Alcohol Adsorption of Poly(N,N-diethylacrylamide) Gel in Water/Alcohol Mixtures, Macromolecules, 48, 11261133, https://doi.org/10.1021/ma502393z, 2015.

Liu, X. J., Li, H. Q., Zhang, B. Y., Wang, Y. J., Ren, X. Y., Guan, S., and Gao, G. H.: Highly stretchable and tough pH-sensitive hydrogels with reversible swelling and recoverable deformation, RSC Adv., 6, 4850-4857, https://doi.org/10.1039/c5ra24414e, 2016.

Mukae, K., Sakurai, M., Sawamura, S., Makino, K., Kim, S. W., Ueda, I., and Shirahama, K.: Swelling of poly(N-isopropylacrylamide) gels in water-alcohol (C1-C4) mixed solvents, J. Phys. Chem., 97, 737-741, https://doi.org/10.1021/j100105a034, 1993.

Nishiyama, Y. and Satoh, M.: Swelling behavior of poly(acrylic acid) gels in aqueous ethanol - effects of counterion species and ionic strength, Macromol. Rapid Comm., 21, 174-177, https://doi.org/10.1002/(SICI)15213927(200003)21:4<174::AID-MARC174>3.0.CO;2-K, 2000.

Osorio, D., Pérez-Correa, J. R., Agosin, E., and Cabrera, M.: Soft-sensor for on-line estimation of ethanol concentrations in wine stills, J. Food Eng., 87, 571-577, https://doi.org/10.1016/j.jfoodeng.2008.01.011, 2008.

Richter, A.: Quellfähige Polymernetzwerke als Aktor-SensorSysteme für die Fluidtechnik, Ph.D. thesis, Technische Universität Dresden, Germany, 2002.

Schmidt, U., Jorsch, C., Guenther, M., and Gerlach, G.: Biochemical piezoresistive sensors based on hydrogels for biotechnology and medical applications, J. Sens. Sens. Syst., 5, 409-417, https://doi.org/10.5194/jsss-5-409-2016, 2016.

Smallwood, I. M. (Eds.): Handbook of organic solvent properties, Arnold, A member of the Hodder Headline Group, London, United Kingdom, 1996.

Wack, H.: Zum Quellungsdruck von polymeren Hydrogelen, Ph.D. thesis, Universität Duisburg-Essen, Germany, 2006.

Winnik, F. M., Ottaviani M. F., Bossmann, S. H., GarciaGaribay, M., and Turro, N. J.: Cononsolvency of Poly(NIsopropylacrylamide) in Mixed Water-Methanol Solutions: A Look at Spin-Labeled Polymers, Macromolecules, 25, 60076017, https://doi.org/10.1021/ma00048a023, 1992. 
$\mathrm{Xu}$, X., Goponenko, A. V., and Asher, S. A.: Polymerized PolyHEMA Photonic Crystals: $\mathrm{pH}$ and Ethanol Sensor Materials, J. Am. Chem. Soc., 130, 3113-3119, https://doi.org/10.1021/ja077979+, 2008. 